\title{
Treatment for intermittent claudication and the effects on walking distance and quality of life
}

Citation for published version (APA):

Kruidenier, L. M., Viechtbauer, W., Nicolai, S. P., Buller, H. R., Prins, M. H., \& Teijink, J. A. W. (2012). Treatment for intermittent claudication and the effects on walking distance and quality of life. Vascular, 20(1), 20-35. https://doi.org/10.1258/vasc.2011.ra0048

Document status and date:

Published: 01/02/2012

DOI:

10.1258/vasc.2011.ra0048

Document Version:

Publisher's PDF, also known as Version of record

Document license:

Taverne

Please check the document version of this publication:

- A submitted manuscript is the version of the article upon submission and before peer-review. There can be important differences between the submitted version and the official published version of record.

People interested in the research are advised to contact the author for the final version of the publication, or visit the DOI to the publisher's website.

- The final author version and the galley proof are versions of the publication after peer review.

- The final published version features the final layout of the paper including the volume, issue and page numbers.

Link to publication

\footnotetext{
General rights rights.

- You may freely distribute the URL identifying the publication in the public portal. please follow below link for the End User Agreement:

www.umlib.nl/taverne-license

Take down policy

If you believe that this document breaches copyright please contact us at:

repository@maastrichtuniversity.nl

providing details and we will investigate your claim.
}

Copyright and moral rights for the publications made accessible in the public portal are retained by the authors and/or other copyright owners and it is a condition of accessing publications that users recognise and abide by the legal requirements associated with these

- Users may download and print one copy of any publication from the public portal for the purpose of private study or research.

- You may not further distribute the material or use it for any profit-making activity or commercial gain

If the publication is distributed under the terms of Article $25 \mathrm{fa}$ of the Dutch Copyright Act, indicated by the "Taverne" license above, 


\title{
Treatment for intermittent claudication and the effects on walking distance and quality of life
}

\author{
L M Kruidenier*, W Viechtbauer ${ }^{\dagger}, S$ P Nicola ${ }^{\ddagger}, H$ Büller ${ }^{\S}, M H$ Prins $^{* *}$ and J A W Teijink ${ }^{\dagger \dagger}$
}

The objective of the study was to provide an overview of the most common treatments for intermittent claudication and to determine the effectiveness in improving walking distance and quality of life based on a combination of direct and indirect evidence. We included trials that compared: angioplasty, surgery, exercise therapy or no treatment for intermittent claudication. Outcome measurements were walking distance (maximum, pain-free) and quality of life (physical, mental). We used a network meta-analysis model for the combination of direct and indirect evidence. We included 42 studies, presenting 3106 participants. The network metaanalysis showed that supervised exercise therapy $(\Delta=1.62, P<0.01)$, angioplasty $(\Delta=1.89, P<0.01)$ and surgery $(\Delta=2.72, P=0.02)$ increased walking distance significantly more than no treatment. Furthermore, supervised exercise therapy $(\Delta=0.60, P<0.01)$, angioplasty $(\Delta=0.91, P=0.01)$ and surgery $(\Delta=1.07, P<0.01)$ increased physical quality of life more than no treatment. However, in the sensitivity analysis, only supervised exercise therapy had additional value over no symptomatic treatment $(\Delta=0.66, P<0.01)$. In conclusion, this network meta-analysis indicates that supervised exercise therapy is more effective in both increasing walking distance and physical quality of life, compared with no treatment. Angioplasty and surgery also increase walking distance, compared with no treatment, but results for physical quality of life are less convincing.

Key words: intermittent claudication; exercise therapy; percutaneous vascular intervention; bypass surgery

\section{Introduction}

Intermittent claudication is a symptom of peripheral arterial disease and is described as an uncomfortable or painful feeling in the calves, buttocks or thighs that is experienced while walking and that is relieved by a short rest. ${ }^{1}$ Survival of patients with intermittent claudication is decreased, mainly due to cardiac and cerebrovascular co-morbidity; ${ }^{2}$ however, the prognosis of the leg is favorable. ${ }^{3,4}$ In the vast majority of cases, intermittent claudication is not a limbthreatening condition. Nevertheless, it has a considerable negative influence on the patient's functional capacity,

\section{Accepted June 2, 2011}

*Department of Surgery, Orbis Medical Centre, Sittard; ${ }^{\dagger}$ Department of Statistics, Maastricht University, Maastricht; ${ }^{*}$ Department of Surgery, Maxima Medical Centre, Eindhoven; ${ }^{\circledR}$ Department of Internal Medicine, Academic Medical Centre, Amsterdam; **Department of Epidemiology, Maastricht University, Maastricht; ${ }^{\dagger}$ Department of Surgery, Catharina Hospital, Eindhoven, The Netherlands

Correspondence to: Joep A W Teijink, Department of Surgery, Catharina Hospital, Michelangelolaan 2, 5623 EJ Eindhoven, The Netherlands; e-mail: joep.teijink@catharina-ziekenhuis.nl ambulatory activity and quality of life. ${ }^{5-7}$ Therefore, treatment of intermittent claudication aims for functional improvement and increase in quality of life rather than limb-salvage.

Exercise therapy is the oldest suggested therapy for intermittent claudication. ${ }^{8}$ More recently, surgical $^{9}$ and endovascular interventions ${ }^{10}$ were described. Traditionally, studies evaluating invasive interventions for intermittent claudication mainly focused on results of increased blood flow rather than functional improvement and changes in quality of life. However, objective indices of peripheral arterial disease (e.g. ankle-brachial index, angiographic extent of the disease) only correlate weakly with functional capacity and quality of life. ${ }^{11}$ More importantly, correlations between hemodynamic improvements and improvements in functional capacity, quality of life and symptoms are only modest. ${ }^{12-14}$ This indicates that outcome measurements other than hemodynamics would be more valuable to describe the results of treatment for intermittent claudication. Furthermore, as was recently described by Cao and De Rango, ${ }^{15}$ the discussion about the best treatment of intermittent claudication is ongoing and lack of evidence of more recent developed techniques was underlined. 
The objective of this meta-analysis is to provide an overview of evidence of the most frequently used treatments for intermittent claudication and to determine their effectiveness in improving walking distance and quality of life.

\section{Materials and methods}

\section{Selection criteria}

All clinical trials that compared at least two of the following treatments for intermittent claudication: angioplasty, surgery, exercise therapy or no treatment were potentially eligible for inclusion in the meta-analysis. Additional inclusion criteria were presence of intermittent claudication (Fontaine stage 2/Rutherford stages 1-3), walking distance (assessed on a treadmill) and/or quality of life as an outcome measurement.

Exclusion criteria were a follow-up duration of less than three months and stages of peripheral arterial disease other than intermittent claudication. Furthermore, studies in languages other than English, German or Dutch were excluded.

\section{Search strategy}

We conducted a search in Pubmed and Embase until February 2009 with the following search terms: peripheral arterial disease, intermittent claudication, peripheral arterial obstructive disease, peripheral vascular disease, peripheral arterial occlusive disease, PAD and PAOD; angioplasty, exercise, walking, endarterectomy and bypass; and quality of life, QOL, short form 36, SF-36, Rand 36, euroqol, sickness impact profile, SIP, vascuqol and walking distance. The reference lists of relevant articles were hand-searched for additional studies.

One author (LMK) selected potentially eligible trials based on the abstracts. These studies were then independently assessed for suitability by two additional authors (LMK, JAWT). If necessary, a third reviewer (MHP) was asked for conclusive advice.

\section{Interventions}

Interventions were categorized into no treatment, nonsupervised exercise therapy (non-SET), supervised exercise therapy (SET), angioplasty, surgery, conservative treatment or invasive treatment by two reviewers (LMK, MHP).

No treatment was defined as no treatment or the use of placebo tablets with or without treatment of vascular risk factors and without any advice to exercise. Non-SET was defined as a single or repeated advice and/or information on exercise. Supervision of exercise was considered to be present if a nurse or physiotherapist was present during the training sessions. Within the definition of angioplasty, both angioplasty alone and angioplasty with (primary or selective) stent placement or laser-assisted angioplasty were accepted. The surgical category consisted of bypass surgery as well as endarterectomy.

Conservative and invasive treatments are categories with mixed treatment modalities. Studies for which it was not clear as to what the exact treatment was or no differentiation was made between the various invasive or conservative treatments were categorized in a mixed category (e.g. angioplasty and surgery).

\section{Outcome measurements}

The primary outcome measurement was the absolute claudication distance (maximum walking distance) as measured by a treadmill test. A progressive treadmill test (constant speed, increasing slope) was preferred, but if not available a continuous treadmill test (constant speed and slope) was an alternative.

Secondary outcome measurements were the initial claudication distance (pain-free walking distance) and quality of life. The preferred quality-of-life assessment was the medical outcomes study 36-item short-form health survey (SF-36), but other quality-of-life instruments were also eligible. The SF-36 was summarized into a physical health component and a mental health component. ${ }^{16}$ Alternative quality-of-life questionnaires were also summarized into physical and mental health components.

\section{Assessment of quality of the reports}

One reviewer assessed (LMK) and a second confirmed (SPN) the quality of each trial according to the criteria of Jadad et al. ${ }^{17}$ Double blinding was not considered, because in trials comparing conservative and invasive treatments blinding is not possible. We gave each trial a summary score ranging from 0 to 3 .

\section{Data extraction and calculation}

If available, from each treatment arm of each study, we extracted the number of patients, baseline standard deviation, baseline mean and follow-up mean for walking distance (absolute and initial claudication distance) and quality of life (mental and physical health). Data of the last 
assessment in the study were used for the analyses. If these data were not available authors were contacted. If authors did not respond to our request or did not have the requested data available, the data were, if possible, calculated based on other information presented in the articles using various methods. ${ }^{18} \mathrm{~A}$ description of the calculations and methods used can be found in Appendix 1.

\section{Statistical analysis}

For all outcome measurements, the standardized mean change between the baseline and follow-up assessment for each treatment arm in the individual studies was calculated (i.e. mean at follow-up minus baseline mean, divided by the baseline standard deviation).

Direct evidence means a direct comparison of different treatments in a (randomized) controlled trial. Indirect evidence means that there is no trial that directly compares the different treatments. However, if two trials compare, for example, treatment $\mathrm{B}$ with, respectively, treatment A and $\mathrm{C}$, something can be said about the effectiveness of $\mathrm{A}$ versus $C$. For example, if treatment $A$ is more effective than treatment $\mathrm{B}$ and treatment $\mathrm{B}$ is more effective than treatment $\mathrm{C}$, a logical conclusion is that treatment $\mathrm{A}$ is more effective than treatment $\mathrm{C}$ although no direct comparison is made.

The direct evidence about the relative effectiveness of one intervention compared with another was then expressed in terms of the difference between the standardized mean change values. These effect sizes were then meta-analyzed based on a random-effects model using restricted maximum-likelihood estimation when at least three effect size estimates were available (with only two estimates, a fixed-effects model was used).

For the combination of the direct and indirect evidence, we used an arm-based network meta-analysis model $^{19}$ for each outcome measure (the network of evidence for the mental health component of quality of life yielded two disconnected sets of interventions, which were examined separately). A single parameter for the random effects variance and a constant correlation of 0.5 between the random effects within a study was assumed, so that the amount of heterogeneity between all pair-wise intervention contrasts was constrained to be equal. ${ }^{20}$ The models were fitted with restricted maximum-likelihood estimation. Based on the fitted models, a relative intervention effectiveness can then be obtained for any two interventions connected with each other via the network of evidence, even if no direct evidence is available to compare those two treatments. For the network meta-analysis, we also conducted sensitivity analyses, leaving out all studies with Jadad scores below 2 (all non-randomized trials).

\section{Results}

One hundred forty-three potential eligible articles were identified up to September 2008. We had to exclude 96 articles for the following reasons: no intermittent claudication $(n=9)$; cohort study evaluating just one treatment $(n=29)$; other treatments described than those included in the meta-analysis $(n=10)$; no treatment specified $(n=15)$; outcome measurement other than walking distance or quality of life $(n=13)$; duplicate publications $(n=10)$; follow-up less than three months $(n=7)$; and other reasons $(n=3)$, leaving 42 studies reported in 47 articles for inclusion in the meta-analysis. References of the excluded articles are listed in Appendix 2.

\section{Description of the studies}

The main characteristics of the included studies are presented in Table 1. Thirty-six articles reported results of randomized controlled trials, four articles concerned controlled trials and seven studies were cohort studies, presenting the results of a total of 3106 participants (no treatment 504, non-SET 531, SET 982, angioplasty 333, surgery 198, conservative 341 and invasive 217).

\section{Absolute claudication distance}

Data for the absolute claudication distance at the end of the study were available for 35 studies. ${ }^{8,921-53}$ The majority of the included trials compared SET with either non-SET $(n=14)$ or no treatment $(n=15)$.

Figure 1a presents the results using only direct evidence. The most obvious result is that SET is on average better in increasing the absolute claudication distance than both non-SET $(P<0.01)$ and no treatment $(P<0.01)$ based on 14 and 15 studies, respectively. The combined direct and indirect evidence is presented in Figure $2 \mathrm{a}-$ results for SET are robust. Furthermore, the combined analysis suggests that angioplasty and surgery increase absolute claudication distance more than non-SET and no treatment, although the comparison of surgery with non-SET just failed to reach statistical significance $(P=0.06)$.

In the sensitivity analysis, 14 studies were excluded. Six of the excluded studies compared non-SET with SET and eight studies compared no treatment with SET. Combining direct and indirect evidence, the results for all comparisons remained. Results of the sensitivity analysis are presented in Table 2. 
Table 1 Characteristics of the included studies

\begin{tabular}{|c|c|c|c|c|c|c|c|c|}
\hline Study & Design & $\begin{array}{l}\text { Jahad } \\
\text { points }\end{array}$ & Treatment & $\mathrm{N}$ & $\begin{array}{l}\text { Loss to } \\
\text { follow-up }\end{array}$ & $\begin{array}{l}\text { Follow-up } \\
\text { (months) }\end{array}$ & Outcome & Treadmill protocol \\
\hline Aquarius $^{55}$ & Cohort & 1 & $\begin{array}{l}\text { Non-SET } \\
\text { Invasive }\end{array}$ & $\begin{array}{r}107 \\
77\end{array}$ & $22 \%$ & 12 & SF-36 & $\mathrm{X}$ \\
\hline Brothers ${ }^{56}$ & Cohort & 0 & $\begin{array}{l}\text { Conservative } \\
\text { Surgery } \\
\text { Angioplasty }\end{array}$ & $\begin{array}{r}77 \\
30 \\
6\end{array}$ & NA & 12 & SF-36 & $\mathrm{X}$ \\
\hline Cheetham $^{21}$ & RCT & 3 & SET & 30 & $7 \%$ & 12 & $\begin{array}{l}\text { ACD } \\
\text { SF-36 }\end{array}$ & $3.5 \mathrm{~km} /$ hour, $12 \%$ \\
\hline Collins $^{57}$ & $\mathrm{RCT}$ & 2 & $\begin{array}{l}\text { No treatment } \\
\text { SET }\end{array}$ & $\begin{array}{l}25 \\
27\end{array}$ & $12 \%$ & 6 & SF-36 & $\mathrm{X}$ \\
\hline Crowther ${ }^{22}$ & RCT & 1 & $\begin{array}{l}\text { Non-SET } \\
\text { SET }\end{array}$ & 11 & NA & 12 & $\mathrm{ACD}, \mathrm{ICD}$ & $\begin{array}{l}3.2 \mathrm{~km} / \text { hour, every } 2 \text { minutes } 2 \% \\
\text { increase }\end{array}$ \\
\hline Currie $^{58}$ & Cohort & 1 & $\begin{array}{l}\text { Non-SET } \\
\text { Angioplasty } \\
\text { Surgery }\end{array}$ & $\begin{array}{l}78 \\
74 \\
34\end{array}$ & $8 \%$ & 3 & SF-36 & $\mathrm{X}$ \\
\hline Dahllöf ${ }^{23}$ & RCT & 1 & $\begin{array}{l}\text { No treatment } \\
\text { SET }\end{array}$ & $\begin{array}{r}8 \\
10\end{array}$ & NA & 6 & $\mathrm{ICD}, \mathrm{ACD}$ & $4.0 \mathrm{~km} /$ hour, $0 \%$ \\
\hline Dahllöf $^{24}$ & RCT & 1 & $\begin{array}{l}\text { No treatment } \\
\text { SET }\end{array}$ & 11 & $29 \%$ & 12 & $\mathrm{ICD}, \mathrm{ACD}$ & $\begin{array}{l}4.0 \mathrm{~km} / \text { hour, } 0 \% \text {. After } 10 \text { minutes } \\
6.0 \mathrm{~km} \text { /hour }\end{array}$ \\
\hline Degischer $^{25}$ & CT & 1 & $\begin{array}{l}\text { Non-SET } \\
\text { SET }\end{array}$ & 23 & $14 \%$ & 6 & $\mathrm{ICD}, \mathrm{ACD}$ & $\begin{array}{l}3.2 \mathrm{~km} / \text { hour, every } 2 \text { minutes } 2 \% \\
\text { increase }\end{array}$ \\
\hline Feinglass $^{59}$ & Cohort & 1 & $\begin{array}{l}\text { Conservative } \\
\text { Surgery } \\
\text { Angioplasty }\end{array}$ & $\begin{array}{r}145 \\
60 \\
44\end{array}$ & $18 \%$ & 18 & SF-36 & $\mathrm{X}$ \\
\hline Gardner $^{26}$ & RCT & 2 & $\begin{array}{l}\text { No treatment } \\
\text { SET }\end{array}$ & 30 & $49 \%$ & 18 & ICD, ACD & $\begin{array}{l}3.2 \mathrm{~km} / \text { hour, every } 2 \text { minutes } 2 \% \\
\text { increase }\end{array}$ \\
\hline Gardne $e^{60}$ & RCT & 2 & $\begin{array}{l}\text { No treatment } \\
\text { SET }\end{array}$ & $\begin{array}{l}30 \\
31\end{array}$ & $49 \%$ & 6 & SF-36 & $\mathrm{X}$ \\
\hline Gelin $^{27}$ & RCT & 3 & $\begin{array}{l}\text { No treatment } \\
\text { SET } \\
\text { Invasive }\end{array}$ & $\begin{array}{l}88 \\
87\end{array}$ & $15 \%$ & 12 & $\mathrm{ACD}$ & $\begin{array}{l}\text { Unknown speed with increasing } \\
\text { slope }\end{array}$ \\
\hline Gibellini $^{28}$ & RCT & 1 & $\begin{array}{l}\text { No treatment } \\
\text { SET }\end{array}$ & $\begin{array}{l}20 \\
20\end{array}$ & $8 \%$ & 6 & $\mathrm{ICD}, \mathrm{ACD}$ & $3.0 \mathrm{~km} /$ hour, $0 \%$ \\
\hline Regensteiner & RCT & 2 & No treatment & 9 & $24 \%$ & 3 & $\mathrm{ICD}, \mathrm{ACD}$ & $\begin{array}{l}3.2 \mathrm{~km} / \text { hour, every } 3 \text { minutes } 3.5 \% \\
\text { increase }\end{array}$ \\
\hline Hiatt $^{29}$ & RCT & 2 & No treatment & $\begin{array}{l}10 \\
10\end{array}$ & $10 \%$ & 3 & $\mathrm{ICD}, \mathrm{ACD}$ & $\begin{array}{l}3.2 \mathrm{~km} / \text { hour, every } 3 \text { minutes } 3.5 \% \\
\text { increase }\end{array}$ \\
\hline & & & SET & 10 & & & & \\
\hline Hobbs $^{30}$ & RCT & 3 & $\begin{array}{l}\text { Non-SET } \\
\text { SET } \\
\text { Angioplasty }\end{array}$ & $\begin{array}{l}7 \\
7 \\
9\end{array}$ & $4 \%$ & 6 & $\mathrm{ICD}, \mathrm{ACD}$ & $3.0 \mathrm{~km} /$ hour, $10 \%$ \\
\hline Hobbs $^{31}$ & RCT & 1 & $\begin{array}{l}\text { Non-SET } \\
\text { SET }\end{array}$ & $\begin{array}{l}9 \\
9\end{array}$ & NA & 6 & $\mathrm{ICD}, \mathrm{ACD}$ & $3.0 \mathrm{~km} /$ hour, $10 \%$ \\
\hline
\end{tabular}


Table 1 Continued

\begin{tabular}{|c|c|c|c|c|c|c|c|c|}
\hline Study & Design & $\begin{array}{l}\text { Jahad } \\
\text { points }\end{array}$ & Treatment & $\mathrm{N}$ & $\begin{array}{l}\text { Loss to } \\
\text { follow-up }\end{array}$ & $\begin{array}{l}\text { Follow-up } \\
\text { (months) }\end{array}$ & Outcome & Treadmill protocol \\
\hline \multirow[t]{2}{*}{ Hodges $^{32}$} & \multirow[t]{2}{*}{ RCT } & \multirow[t]{2}{*}{2} & Non-SET & 14 & \multirow[t]{2}{*}{ NA } & \multirow[t]{2}{*}{3} & \multirow[t]{2}{*}{$\mathrm{ACD}$} & \multirow[t]{2}{*}{$\begin{array}{l}3.2 \mathrm{~km} / \text { hour, every } 2 \text { minutes } 2 \% \\
\text { increase }\end{array}$} \\
\hline & & & SET & 14 & & & & \\
\hline Holm $^{33}$ & RCT & 1 & $\begin{array}{l}\text { No treatment } \\
\text { SET }\end{array}$ & $\begin{array}{l}6 \\
6\end{array}$ & NA & 4 & ICD, ACD & Unknown \\
\hline Imfeld $^{61}$ & $\mathrm{CT}$ & 1 & $\begin{array}{l}\text { Non-SET } \\
\text { SET }\end{array}$ & $\begin{array}{l}20 \\
18\end{array}$ & $20 \%$ & 6 & SF-36 & $\mathrm{X}$ \\
\hline \multirow[t]{2}{*}{ Izquierdo-Porrera ${ }^{34}$} & \multirow[t]{2}{*}{ Cohort } & \multirow[t]{2}{*}{0} & No treatment & 14 & \multirow[t]{2}{*}{ NA } & \multirow[t]{2}{*}{6} & \multirow[t]{2}{*}{ ICD, ACD } & \multirow[t]{2}{*}{$\begin{array}{l}3.2 \mathrm{~km} / \text { hour, every } 2 \text { minutes } 2 \% \\
\text { increase }\end{array}$} \\
\hline & & & SET & 34 & & & & \\
\hline Jansen $^{35}$ & $\mathrm{RCT}$ & 1 & $\begin{array}{l}\text { No treatment } \\
\text { SET }\end{array}$ & $\begin{array}{l}24 \\
24\end{array}$ & NA & 24 & $\mathrm{ICD}, \mathrm{ACD}$ & $3.5 \mathrm{~km} /$ hour, $10 \%$ \\
\hline Kakkos $^{37}$ & RCT & 3 & Non-SET & 9 & $38 \%$ & 12 & $\begin{array}{l}\text { ICD, ACD, } \\
\text { SF-36 }\end{array}$ & 3.5 km/hour, $10 \%$ \\
\hline & & & SET & 12 & & & & \\
\hline Langbein $^{38}$ & RCT & 2 & No treatment & 25 & $12 \%$ & 6 & $\mathrm{ACD}$ & $\begin{array}{c}3.0 \mathrm{~km} / \text { hour, every } 0.5 \text { minute } 0.5 \% \\
\text { increase. After } 6 \text { minutes every } 3 \\
\text { minutes } 0.32 \mathrm{~km} / \text { hour increase }\end{array}$ \\
\hline & & & SET & 27 & & & & \\
\hline Larsen $^{8}$ & RCT & 2 & $\begin{array}{l}\text { No treatment } \\
\text { Non-SET }\end{array}$ & $\begin{array}{l}7 \\
7\end{array}$ & $0 \%$ & 6 & $\mathrm{ICD}, \mathrm{ACD}$ & $4.6 \mathrm{~km} /$ hour, $0 \%$ or $8 \%$ or $16 \%$ \\
\hline $\mathrm{Lee}^{39}$ & CT & 0 & Non-SET & 37 & NA & 6 & $\begin{array}{l}\text { ICD, ACD } \\
\text { SF-36 }\end{array}$ & $3.8 \mathrm{~km} /$ hour, $10 \%$ \\
\hline & & & SET & 33 & & & & \\
\hline Lundgren ${ }^{9}$ & $\mathrm{RCT}$ & 3 & $\begin{array}{l}\text { SET } \\
\text { Surgery }\end{array}$ & $\begin{array}{l}25 \\
25\end{array}$ & $16 \%$ & 12 & $\mathrm{ICD}, \mathrm{ACD}$ & $4.0 \mathrm{~km} /$ hour, $0 \%$ \\
\hline Mannarino $^{41}$ & CT & 0 & $\begin{array}{l}\text { No treatment } \\
\text { SET }\end{array}$ & $\begin{array}{l}8 \\
8\end{array}$ & NA & 6 & ICD, ACD & $2.0 \mathrm{~km} /$ hour, $12 \%$ \\
\hline Mannarino ${ }^{40}$ & RCT & 1 & $\begin{array}{l}\text { No treatment } \\
\text { SET }\end{array}$ & $\begin{array}{l}10 \\
20\end{array}$ & NA & 6 & $\mathrm{ICD}, \mathrm{ACD}$ & $2.0 \mathrm{~km} /$ hour, $12 \%$ \\
\hline $\mathrm{Mika}^{54}$ & RCT & 2 & $\begin{array}{l}\text { No treatment } \\
\text { SET }\end{array}$ & $\begin{array}{l}49 \\
49\end{array}$ & $18 \%$ & 3 & ICD & $3.2 \mathrm{~km} /$ hour, $12 \%$ \\
\hline Mika $^{42}$ & RCT & 2 & No treatment & 30 & $8 \%$ & 3 & ICD, ACD & $\begin{array}{l}3.2 \mathrm{~km} / \text { hour, every } 3 \text { minutes } 3.5 \% \\
\text { increase }\end{array}$ \\
\hline & & & SET & 30 & & & & \\
\hline Nielsen $^{43}$ & RCT & 3 & $\begin{array}{l}\text { Non-SET } \\
\text { SET }\end{array}$ & $\begin{array}{l}26 \\
25\end{array}$ & $12 \%$ & 3 & ACD & $3.8 \mathrm{~km} /$ hour, $8 \%$ \\
\hline Nylaende $^{44}$ & RCT & 3 & $\begin{array}{l}\text { Non-SET } \\
\text { Angioplasty }\end{array}$ & $\begin{array}{l}28 \\
28\end{array}$ & $14 \%$ & 24 & $\mathrm{ICD}, \mathrm{ACD}$ & $3.0 \mathrm{~km} /$ hour, $10 \%$ \\
\hline Patterson $^{45}$ & RCT & 2 & Non-SET & 30 & $37 \%$ & 6 & $\begin{array}{l}\text { ICD, ACD } \\
\text { SF-36 }\end{array}$ & $\begin{array}{l}1.6 \mathrm{~km} / \text { hour, } 5 \% \text {. Every } 5 \text { minutes } \\
\text { increase in speed and slope }\end{array}$ \\
\hline & & & SET & 30 & & & & \\
\hline Pell $^{62}$ & Cohort & 1 & $\begin{array}{l}\text { Conservative } \\
\text { Surgery } \\
\text { Angioplasty }\end{array}$ & $\begin{array}{r}119 \\
19 \\
19\end{array}$ & $22 \%$ & 6 & SF-36 & $\mathrm{X}$ \\
\hline Perkins ${ }^{46}$ & RCT & 2 & $\begin{array}{l}\text { SET } \\
\text { PTA }\end{array}$ & $\begin{array}{l}26 \\
30\end{array}$ & $34 \%$ & 70 & $\mathrm{ACD}$ & $3.0 \mathrm{~km} /$ hour, $10 \%$ \\
\hline
\end{tabular}


Table 1 Continued

\begin{tabular}{|c|c|c|c|c|c|c|c|c|}
\hline Study & Design & $\begin{array}{l}\text { Jahad } \\
\text { points }\end{array}$ & Treatment & $\mathrm{N}$ & $\begin{array}{l}\text { Loss to } \\
\text { follow-up }\end{array}$ & $\begin{array}{l}\text { Follow-up } \\
\text { (months) }\end{array}$ & Outcome & Treadmill protocol \\
\hline Regensteiner $^{63}$ & RCT & 2 & $\begin{array}{l}\text { No treatment } \\
\text { SET }\end{array}$ & $\begin{array}{l}10 \\
10\end{array}$ & $10 \%$ & 3 & SF-20 & $\mathrm{X}$ \\
\hline \multirow[t]{2}{*}{ Regensteiner ${ }^{47}$} & RCT & 2 & Non-SET & 10 & $0 \%$ & 3 & $\begin{array}{l}\text { ICD, ACD, } \\
\text { SF-20 }\end{array}$ & $\begin{array}{l}3.2 \mathrm{~km} / \text { hour, every } 3 \text { minutes } 3.5 \% \\
\text { increase }\end{array}$ \\
\hline & & & SET & 10 & & & & \\
\hline \multirow[t]{2}{*}{ Savage ${ }^{48}$} & RCT & 1 & Non-SET & 10 & NA & 6 & $\begin{array}{l}\text { ICD, ACD, } \\
\text { SF-36 }\end{array}$ & $\begin{array}{l}3.2 \mathrm{~km} / \text { hour, every } 2 \text { minutes } 2 \% \\
\text { increase }\end{array}$ \\
\hline & & & SET & 11 & & & & \\
\hline \multirow[t]{2}{*}{ Spronk $^{49}$} & RCT & 3 & SET & 75 & $7 \%$ & 12 & $\begin{array}{l}\text { ICD, ACD, } \\
\text { SF-36 }\end{array}$ & $3.5 \mathrm{~km} /$ hour, $0 \%$ \\
\hline & & & Angioplasty & 75 & & & & \\
\hline \multirow[t]{2}{*}{ Stewart $^{50}$} & RCT & 3 & Non-SET & 26 & $8 \%$ & 6 & ICD, ACD & $2.5 \mathrm{~km} /$ hour, $10 \%$ \\
\hline & & & SET & 29 & & & & \\
\hline \multirow[t]{3}{*}{$\mathrm{Taft}^{64}$} & RCT & 3 & No treatment & 57 & $32 \%$ & 12 & SIP & $\mathrm{X}$ \\
\hline & & & SET & 61 & & & & \\
\hline & & & Invasive & 53 & & & & \\
\hline \multirow[t]{2}{*}{$\mathrm{Tisi}^{51}$} & RCT & 1 & Non-SET & 17 & NA & 12 & $\mathrm{ICD}, \mathrm{ACD}$ & $3.0 \mathrm{~km} /$ hour, $10 \%$ \\
\hline & & & SET & 22 & & & & \\
\hline \multirow[t]{2}{*}{ Tsai $^{52}$} & RCT & 2 & No treatment & 32 & $17 \%$ & 3 & $\begin{array}{l}\text { ICD, ACD, } \\
\text { SF-36 }\end{array}$ & $\begin{array}{l}3.2 \mathrm{~km} / \text { hour, every } 2 \text { minutes } 2 \% \\
\text { increase }\end{array}$ \\
\hline & & & SET & 32 & & & & \\
\hline \multirow[t]{2}{*}{ Wann-Hansson ${ }^{65}$} & Cohort & 1 & Angioplasty & 18 & $21 \%$ & 12 & SF-36 & $\mathrm{X}$ \\
\hline & & & Surgery & 30 & & & & \\
\hline \multirow[t]{2}{*}{ Whyman $^{53}$} & RCT & 3 & Non-SET & 32 & $11 \%$ & 24 & ICD, ACD & $4.0 \mathrm{~km} /$ hour, $10 \%$ \\
\hline & & & Angioplasty & 30 & & & & \\
\hline
\end{tabular}

Non-SET, non-supervised exercise therapy; SET, supervised exercise therapy; RCT, randomized controlled trial; CT, controlled trial; NA, not available; SF, short form; ACD, absolute claudication distance; ICD, initial claudication distance; SIP, sickness impact profile

\section{Initial claudication distance}

Data from 30 studies, reporting 32 comparisons on the initial claudication distance, were available (Figure 1b). ${ }^{8,9,22-26,28-31,33-37,39-42,44,45,47-54}$ Most trials compared SET with non-SET $(n=11)$ or SET with no treatment $(n=14)$.

The most obvious result is that SET increases initial claudication distance more than both no treatment and non-SET. The results based on only the direct comparisons for initial claudication distance are depicted in Figure $1 \mathrm{~b}$.

Combining both direct and indirect evidence, SET, angioplasty and surgery are more effective when compared with no treatment in increasing initial claudication distance. Additionally, surgery performs better than non-SET, SET and angioplasty.

In the sensitivity analyses (Table 2), 14 studies were excluded. Six of the excluded studies compared non-SET with SET and eight studies compared no treatment with
SET. Combining direct and indirect evidence, SET, angioplasty and surgery remain better in improving initial claudication distance compared with no treatment. Surgery is still more effective than non-SET, SET and angioplasty, although the comparison with angioplasty just failed to reach statistical significance $(P=0.06)$.

\section{Physical health}

Data from 19 studies reported 29 direct comparisons for physical health (Figure 1c). ${ }^{21,37,39,45,47-49,52,55-65}$ Using only direct evidence, SET increases physical health more than no treatment $(P=0.01)$. Other direct results for physical health are presented in Figure 1c.

Combining direct and indirect evidence, SET, angioplasty and surgery are more effective in improving physical health when compared with no treatment. Furthermore, angioplasty and surgery are more effective than both 
non-SET and conservative treatment. The results of the combined direct and indirect evidence are presented in Figure 2c.
For the sensitivity analysis, eight studies were excluded, leaving 11 studies describing 12 direct comparisons. SET was compared with no treatment $(n=5)$, non-SET $(n=4)$,

(a)

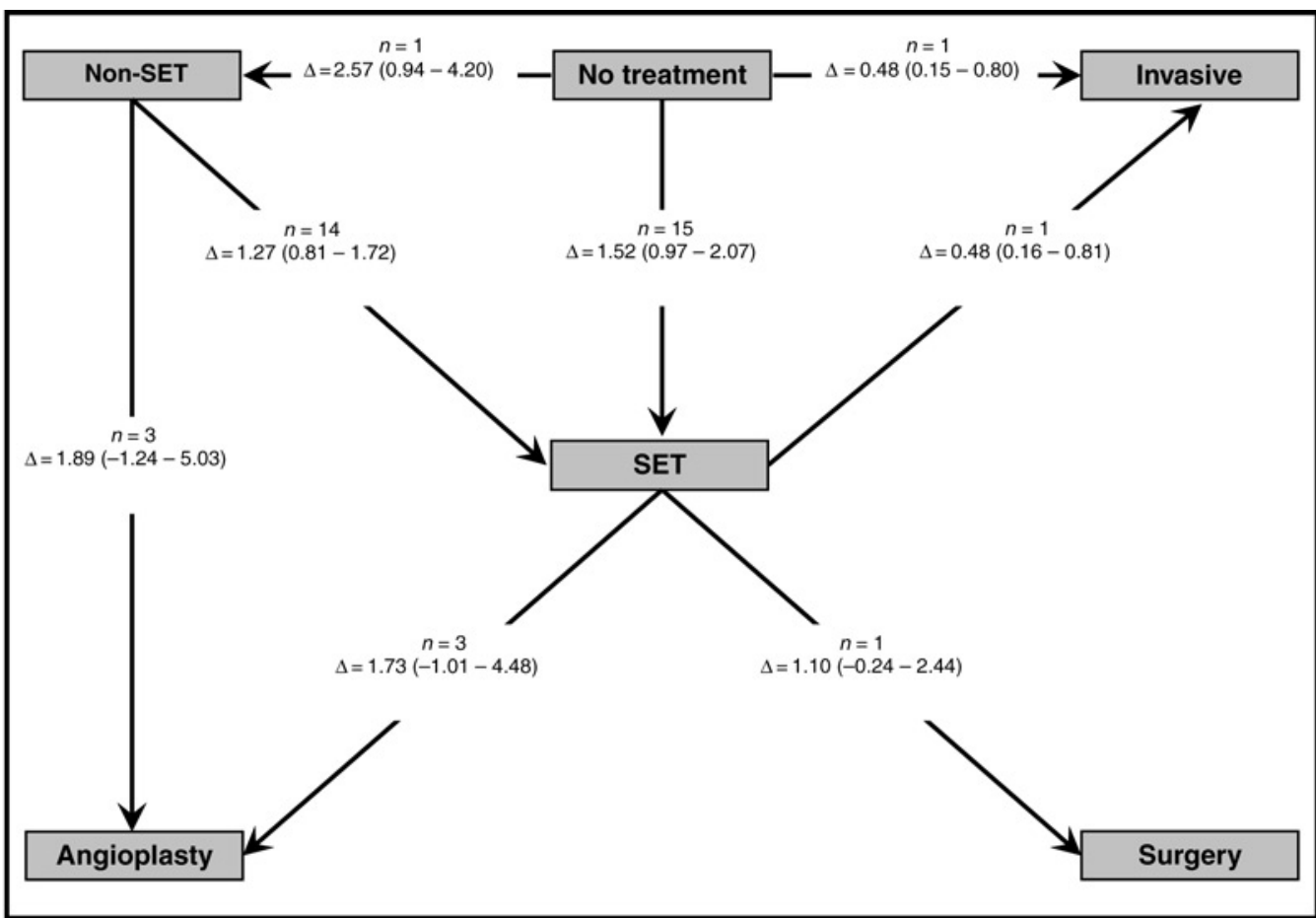

(b)

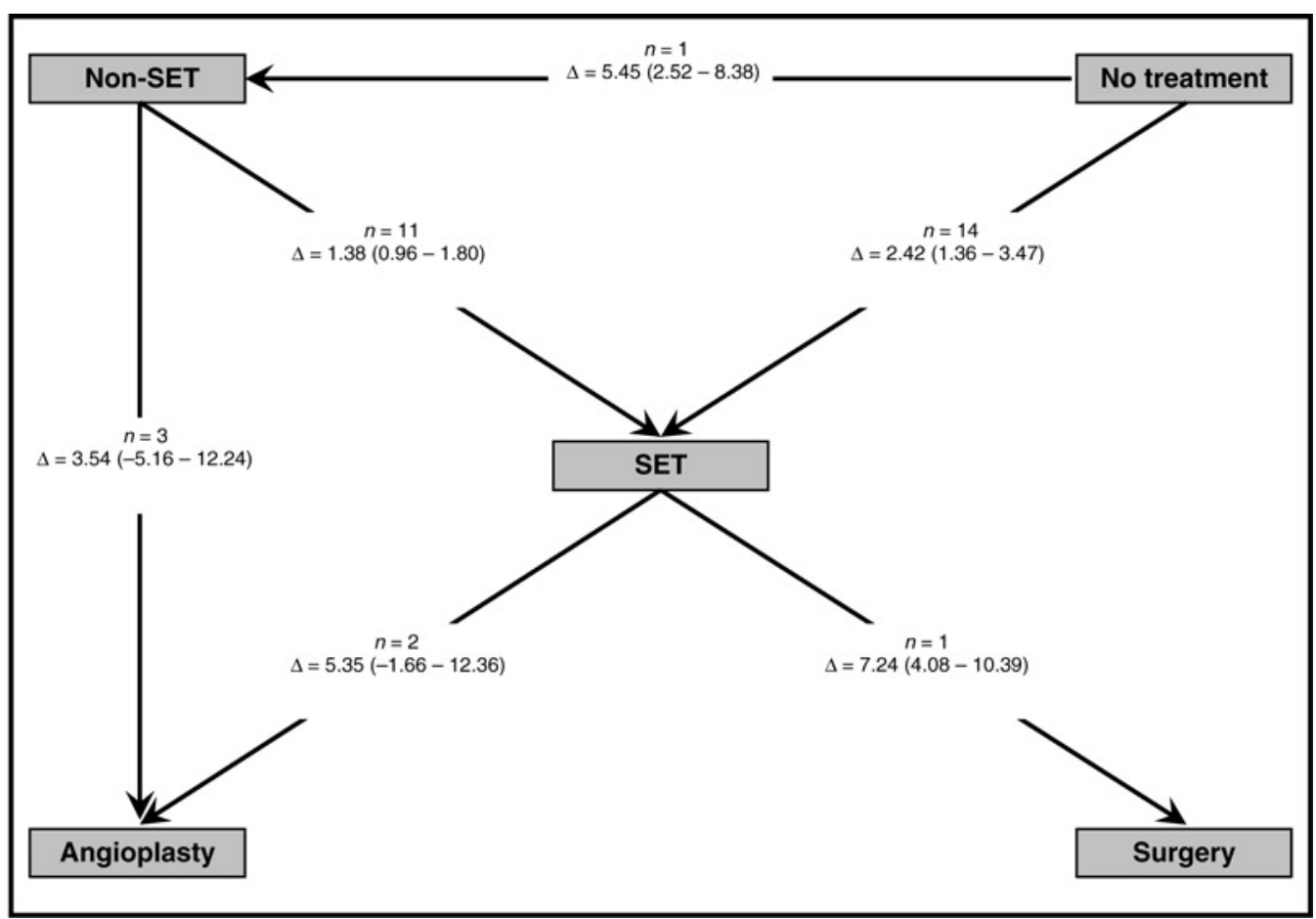

Figure 1 Continued 
(c)

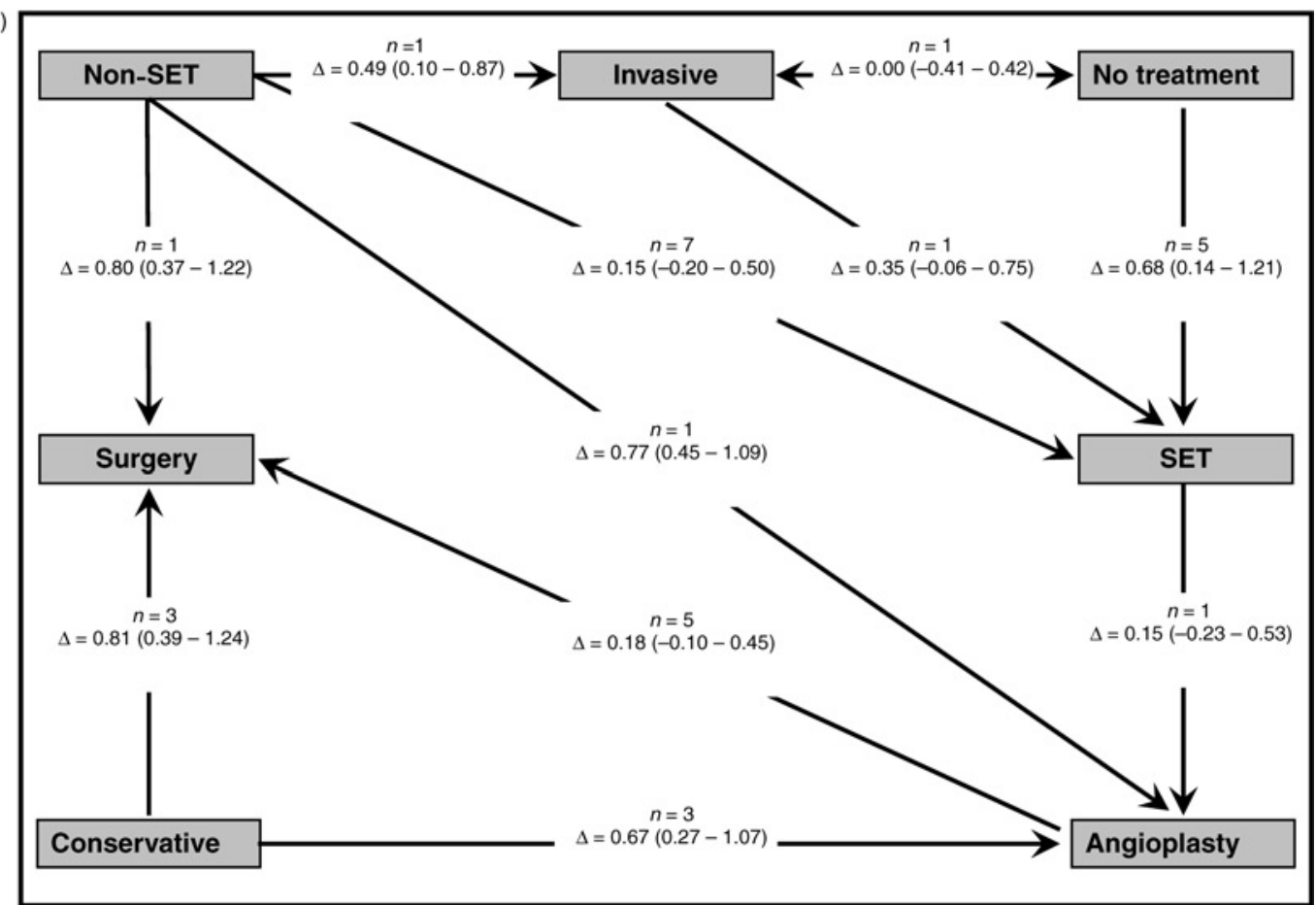

Figure 1 Results of the direct comparisons. In this figure only direct evidence is gathered for all outcome measurements. In the gray blocks, the different treatments are indicated. In the white blocks, the number of studies $(n)$, the difference in standardized change $(\Delta)$ with a $95 \%$ confidence interval, and the $P$ value are noted. The arrows indicate the direction of the effect and the head of the arrow points to the more effective treatment. (a), (b) and (c) describe the results of the absolute claudication distance, initial claudication distance and physical health. Non-SET, non-supervised exercise therapy; SET, supervised exercise therapy

angioplasty $(n=1) \quad$ and invasive treatment $\quad(n=1)$. difference in standardized change of $0.66(95 \%$ CI 0.20 Furthermore, invasive treatment was compared with no 1.13). All other treatments are not significantly different treatment. In the sensitivity analysis, the results of SET from each other, or comparisons are excluded from the compared with no treatment remain in favor of SET with a analysis as part of the sensitivity analysis.

(a)

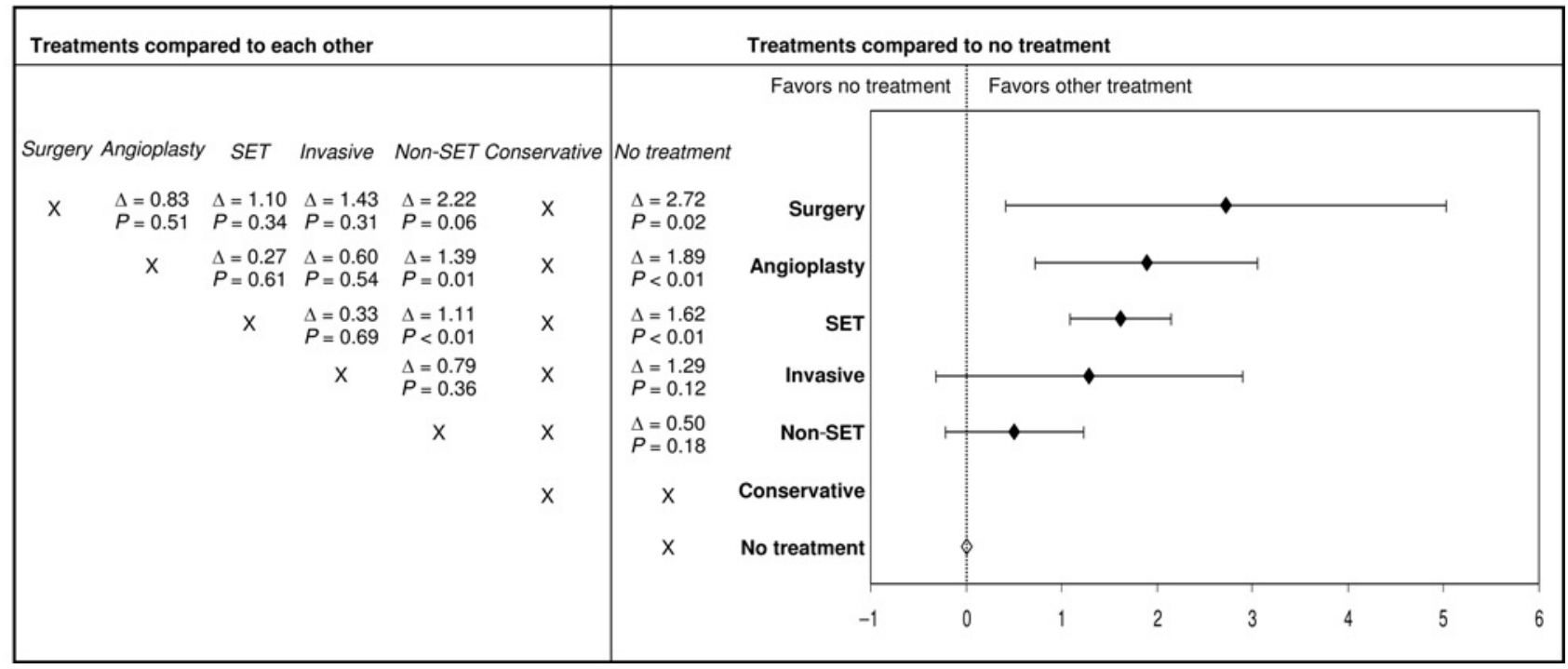

Figure 2 Continued 
(b)

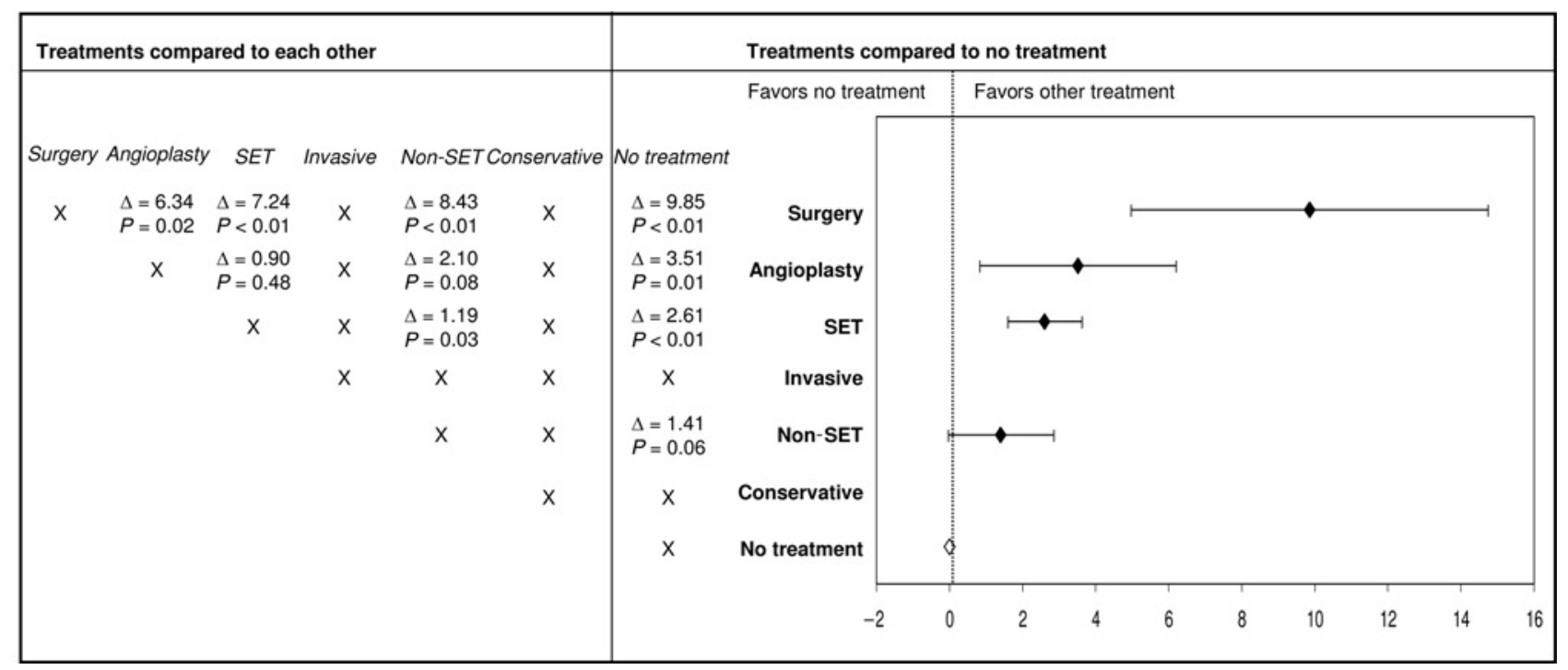

(c)

\begin{tabular}{|c|c|c|c|c|c|c|c|c|c|c|}
\hline \multicolumn{6}{|c|}{ Treatments compared to each other } & \multicolumn{4}{|c|}{ Treatments compared to no treatment } & \\
\hline \multirow{10}{*}{$\begin{array}{r}\text { Surge } \\
\mathrm{x}\end{array}$} & & & & & & \multirow{2}{*}{\multicolumn{2}{|c|}{ No treatment }} & Favors no treatment & Favors other treatment & \\
\hline & Angioplasty & SET & Invasive & Non-SET & Conservative & & & & & \\
\hline & $\begin{aligned} \Delta & =0.16 \\
& =0.45\end{aligned}$ & $\begin{aligned} \Delta & =0.47 \\
& =0.13\end{aligned}$ & $\begin{aligned} \Delta & =0.65 \\
& =0.10\end{aligned}$ & $\begin{aligned} \Delta & =0.76 \\
& =0.01\end{aligned}$ & $\begin{aligned} \Delta & =0.81 \\
& <0.01\end{aligned}$ & $\begin{aligned} \Delta & =1.07 \\
& <0.01\end{aligned}$ & Surgery & & & \\
\hline & \multirow[t]{7}{*}{$\mathrm{x}$} & $\begin{aligned} \Delta & =0.31 \\
& =0.24\end{aligned}$ & $\begin{aligned} \Delta & =0.49 \\
& =0.18\end{aligned}$ & $\begin{aligned} \Delta & =0.60 \\
& =0.02\end{aligned}$ & $\begin{aligned} \Delta & =0.65 \\
& =0.01\end{aligned}$ & $\begin{aligned} \Delta & =0.91 \\
& =0.01\end{aligned}$ & Angioplasty & & $\longmapsto$ & \\
\hline & & $\mathrm{x}$ & $\begin{aligned} \Delta & =0.18 \\
& =0.52\end{aligned}$ & $\begin{aligned} \Delta & =0.28 \\
& =0.09\end{aligned}$ & $\begin{aligned} \Delta & =0.33 \\
& =0.33\end{aligned}$ & $\begin{aligned} \Delta & =0.60 \\
& <0.01\end{aligned}$ & SET & & $\longmapsto$ & \\
\hline & & & $\mathrm{x}$ & $\begin{aligned} \Delta & =0.11 \\
& =0.70\end{aligned}$ & $\begin{aligned} \Delta & =0.16 \\
& =0.70\end{aligned}$ & $\begin{aligned} \Delta & =0.42 \\
& =0.16\end{aligned}$ & Invasive & $\longmapsto$ & $\bullet$ & \\
\hline & & & & $\mathrm{x}$ & $\begin{aligned} \Delta & =0.05 \\
& =0.88\end{aligned}$ & $\begin{aligned} \Delta & =0.31 \\
& =0.22\end{aligned}$ & Non-SET & & -1 & \\
\hline & & & & & $\mathrm{x}$ & $\begin{aligned} \Delta & =0.26 \\
& =0.50\end{aligned}$ & Conservative & & 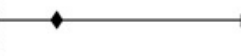 & \\
\hline & & & & & & $\mathrm{x}$ & No treatment & & & \\
\hline & & & & & & & & & 1 & 2 \\
\hline
\end{tabular}

Figure 2 Results of the network meta-analysis. In this figure, both direct and indirect evidence is combined for all outcome measurements. In the right blocks, forests plots are shown that compare the treatments with no symptomatic treatment as reference. Results are indicated as difference in standardized change $(\Delta)$ with a $95 \%$ confidence interval. In the left blocks, the other treatments are compared with each other. Results are indicated as the difference in standardized change $(\Delta)$ with the $P$ value. A positive $\Delta$ indicates that the treatment modality in the column (bold) is superior to the treatment modality in the row (cursive). A negative $\Delta$ indicates the opposite. (a), (b) and (c) describe the results of the absolute claudication distance, initial claudication distance and physical health, respectively. Non-SET, non-supervised exercise therapy; SET, supervised exercise therapy

\section{Mental health}

Data from 14 studies reported 20 direct comparisons for mental health. ${ }^{37,39,45,47,48,52,55-57,60,62-65}$ None of the included treatments differed significantly from each other in the direct, indirect and sensitivity analysis (data not shown).

\section{Discussion}

This network meta-analysis shows that SET, angioplasty and surgery are more beneficial in increasing pain-free and maximum walking distance as compared with no treatment. For physical health, SET has an additional benefit compared with no treatment. For angioplasty and surgery, 
Table 2 Results of the sensitivity analyses*

\begin{tabular}{|c|c|c|c|}
\hline & $\begin{array}{l}\text { Absolute } \\
\text { claudication } \\
\text { distance }\end{array}$ & $\begin{array}{l}\text { Initial } \\
\text { claudication } \\
\text { distance }\end{array}$ & $\begin{array}{l}\text { Physical } \\
\text { health } \\
\text { component }\end{array}$ \\
\hline Surgery & $\begin{array}{l}\Delta=2.84(0.17 \\
\quad \text { to } 5.50)\end{array}$ & $\begin{array}{c}\Delta=10.4(4.86 \\
\quad \text { to } 15.95)\end{array}$ & $\mathrm{X}$ \\
\hline Angioplasty & $\begin{array}{l}\Delta=2.18(0.72 \\
\quad \text { to } 3.65)\end{array}$ & $\begin{array}{c}\Delta=4.55(1.23 \\
\quad \text { to } 7.88)\end{array}$ & $\begin{array}{c}\Delta=0.81 \\
\quad(-0.21 \text { to } \\
1.84)\end{array}$ \\
\hline SET & $\begin{array}{c}\Delta=1.74(0.89 \\
\quad \text { to } 2.58)\end{array}$ & $\begin{array}{l}\Delta=3.16(1.45 \\
\quad \text { to } 4.88)\end{array}$ & $\begin{array}{c}\Delta=0.66(0.20 \\
\text { to } 1.13)\end{array}$ \\
\hline Invasive & $\begin{array}{l}\Delta=1.35(-0.58 \\
\quad \text { to } 3.27)\end{array}$ & $\mathrm{X}$ & $\begin{array}{c}\Delta=0.16 \\
\quad(-0.68 \text { to } \\
1.00)\end{array}$ \\
\hline Non-SET & $\begin{array}{l}\Delta=0.76(-0.33 \\
\quad \text { to } 1.86)\end{array}$ & $\begin{array}{l}\Delta=2.38(0.04 \\
\quad \text { to } 4.73)\end{array}$ & $\begin{array}{c}\Delta=0.29 \\
\quad(-0.46 \text { to } \\
1.04)\end{array}$ \\
\hline Conservative & $\mathrm{X}$ & $\mathrm{X}$ & $\mathrm{X}$ \\
\hline
\end{tabular}

SET, supervised exercise therapy; non-SET, non-supervised exercise therapy

*Data are presented as difference in standardized change ( $\delta$ ) and $95 \%$ confidence interval. All data shown use no symptomatic treatment as referent treatment

improvements in physical health compared with no treatment are seen in the combined analysis. However, in the sensitivity analysis this was not a robust result. None of the studied treatments had any effect on mental health.

Moreover, no obvious differences in effectiveness were found when comparing SET, angioplasty and surgery with each other. Only for increasing pain-free walking distance, surgery seems more effective than SET.

Watson et al. ${ }^{66}$ compared exercise therapy with other treatments and concluded that compared with no treatment, exercise therapy significantly improved maximum walking distance. Evidence for exercise compared with other treatments was limited.

A meta-analysis of Fowkes and Gillespie ${ }^{67}$ compared angioplasty with non-surgical management. Two studies are included in this meta-analysis and with the non-SET-based study results, angioplasty was significantly better than nonsurgical management. However, as in our meta-analysis, with the SET-based study results, no significant difference was found between angioplasty and non-surgical management. Spronk et al. ${ }^{14}$ compared exercise therapy with angioplasty and found that both treatments brought about improvements in physical health-related quality of life. However, no differences between exercise and angioplasty were found.

One meta-analysis compared bypass surgery with other treatments for patients with chronic lower-limb ischemia. ${ }^{68}$
The authors concluded that there was limited evidence for a superior effectiveness of bypass surgery compared with other treatments and that further large trials are required.

The results from the literature mentioned above are in line with the results of our current network meta-analysis.

A striking finding of this meta-analysis is the discrepancy between the number of trials studying the effect of exercise therapy $(n=43)$ compared with the number that studied the effect of angioplasty/surgery $(n=14)$. This imbalance had implications for the sensitivity analyses as almost no studies remained in the analyses to compare angioplasty and surgery with other treatments. Therefore, further research is needed to assess the functional and quality-of-life outcomes of especially angioplasty and surgery.

\section{Limitations}

To establish the effectiveness of an intervention, direct evidence from randomized controlled trials should be used whenever possible. However, due to the limited number of high-quality direct comparisons, indirect evidence was added to the current meta-analysis, bringing uncertainty to our results.

Another potential limitation is the inclusion of several non-randomized and cohort studies in the meta-analysis. Lack of randomization may result in biased effect size estimates, especially when patient characteristics play a role in selecting the treatment. To address this problem, we performed sensitivity analyses that excluded all nonrandomized trials.

Finally, we used end-of-study data as the primary outcome instead of a fixed follow-up time for each study. The reason was that many studies differed in follow-up and that the number of studies was not sufficient to do the analyses for the different follow-up periods separately. Clearly, follow-up time could have an influence on the results of the different interventions (e.g. the time until maximum treatment effect for angioplasty is different than for SET). We examined this issue by including follow-up time as a potential moderator in the meta-analyses of the direct comparisons. However, no moderating effects of follow-up time were found.

According to the current meta-analysis, three treatment options for increasing walking distance in patients with intermittent claudication are effective, namely SET, angioplasty and surgery. However, not only the effectiveness of a treatment, but also the complication risks should be taken into consideration when making a treatment plan for an individual patient. SET is an effective, as well as safe, option 
to treat patients with intermittent claudication given that it is a non-invasive treatment. On the other hand, both angioplasty and bypass surgery are invasive treatment options with known morbidity and mortality. Taking the results from this meta-analysis plus the known complication risks from invasive treatment into account, the clinical implication should be that patients with intermittent claudication should first be treated with supervised exercise therapy. Accordingly, efforts should be made to make supervised exercise programs universally available for patients with intermittent claudication.

In conclusion, supervised exercise therapy is more effective in both increasing walking distance and physical health when compared with no treatment in patients with intermittent claudication. Angioplasty and surgery also increase walking distance, compared with no treatment, but results for physical quality of life are based on mostly nonrandomized trials.

Large randomized controlled trials comparing supervised exercise therapy, angioplasty and bypass surgery in terms of walking distance and quality of life are necessary to establish the potential extra benefits of angioplasty and bypass surgery in the treatment of intermittent claudication.

\section{Conflicts of interest: None.}

\section{References}

1 Norgren L, Hiatt WR, Dormandy JA, Nehler MR, Harris KA, Fowkes FG. Inter-Society Consensus for the Management of Peripheral Arterial Disease (TASC II). J Vasc Surg 2007;45(Suppl S):S5-67

2 Criqui MH, Langer RD, Fronek A, et al. Mortality over a period of 10 years in patients with peripheral arterial disease. $N$ Engl J Med 1992;326:381-6

3 Hirsch AT, Haskal ZJ, Hertzer NR, et al. ACC/AHA Guidelines for the management of patients with peripheral arterial diseases (lower extremity, renal, mesenteric and abdominal aortic): a collaborative report from the American Association for Vascular Surgery/Society for Vascular Surgery, Society for Cardiovascular Angiography and Interventions, Society for Interventional Radiology, Society for Vascular Medicine and Biology, and the American College of Cardiology/American Heart Association Task Force on Practice Guidelines (Writing Committee to Develop Guidelines for the Management of Patients with Peripheral Arterial Disease). Circulation 2005;21:e463-e654

4 Weitz JI, Byrne J, Clagett GP, et al. Diagnosis and treatment of chronic arterial insufficiency of the lower extremities: a critical review. Circulation 1996;94:3026-49

5 Regensteiner JG, Hiatt WR, Coll JR, et al. The impact of peripheral arterial disease on health-related quality of life in the Peripheral Arterial Disease Awareness, Risk, and Treatment: New Resources for Survival (PARTNERS) Program. Vasc Med 2008;13:15-24
6 Gardner AW, Montgomery PS, Scott KJ, Afaq A, Blevins SM. Patterns of ambulatory activity in subjects with and without intermittent claudication. J Vasc Surg 2007;46:1208-14

7 Dumville JC, Lee AJ, Smith FB, Fowkes FG. The health-related quality of life of people with peripheral arterial disease in the community: the Edinburgh Artery Study. Br J Gen Pract 2004;54: 826-31

8 Larsen OA, Lassen NA. Effect of daily muscular exercise in patients with intermittent claudication. Lancet 1966;2:1093-6

9 Lundgren F, Dahllöf AG, Lundholm K, Schersten T, Volkmann R. Intermittent claudication - surgical reconstruction or physical training? A prospective randomized trial of treatment efficiency. Ann Surg 1989;209:346-55

10 Dotter CT, Judkins MP. Transluminal treatment of arteriosclerotic obstruction. Description of a new technic and a preliminary report of its application. Circulation 1964;30:654-70

11 Muller-Buhl U, Engeser P, Klimm HD, Wiesemann A. Quality of life and objective disease criteria in patients with intermittent claudication in general practice. Fam Pract 2003;20:36-40

12 Regensteiner JG, Hargarten ME, Rutherford RB, Hiatt WR. Functional benefits of peripheral vascular bypass surgery for patients with intermittent claudication. Angiology 1993;44:1-10

13 Reekers JA, Vorwerk D, Rousseau H, et al. Results of a European multicentre iliac stent trial with a flexible balloon expandable stent. Eur J Vasc Endovasc Surg 2002;24:511-5

14 Spronk S, Bosch JL, Veen HF, den Hoed PT, Hunink MG. Intermittent claudication: functional capacity and quality of life after exercise training or percutaneous transluminal angioplastysystematic review. Radiology 2005;235:833-42

15 Cao P, De Rango P. Endovascular treatment of peripheral artery disease (PAD): so old yet so far from evidence! Eur J Vasc Endovasc Surg 2009;37:501-3

16 Ware JE Jr, Kosinski M, Bayliss MS, McHorney CA, Rogers WH, Raczek A. Comparison of methods for the scoring and statistical analysis of SF-36 health profile and summary measures: summary of results from the Medical Outcomes Study. Med Care 1995;33: AS264-79

17 Jadad AR, Moore RA, Carroll D, et al. Assessing the quality of reports of randomized clinical trials: is blinding necessary? Control Clin Trials 1996;17:1-12

18 Higgins JPT, Green S. Cochrane Handbook for Systematic Reviews of Interventions Version 5.0.1 [updated September 2008]. The Cochrane Collaboration 2008. See www.cochrane-handbook.org (last checked 29 June 2009)

19 Salanti G, Higgins JP, Ades AE, Ioannidis JP. Evaluation of networks of randomized trials. Stat Methods Med Res 2008;17:279-301

$20 \mathrm{Lu} \mathrm{G}$, Ades AE. Combination of direct and indirect evidence in mixed treatment comparisons. Stat Med 2004;23:3105-24

21 Cheetham DR, Burgess L, Ellis M, Williams A, Greenhalgh RM, Davies AH. Does supervised exercise offer adjuvant benefit over exercise advice alone for the treatment of intermittent claudication? A randomised trial. Eur J Vasc Endovasc Surg 2004; 27:17-23

22 Crowther RG, Spinks WL, Leicht AS, Sangla K, Quigley F, Golledge J. Effects of a long-term exercise program on lower limb mobility, physiological responses, walking performance, and physical activity levels in patients with peripheral arterial disease. J Vasc Surg 2008;47:303-9

23 Dahllöf AG, Bjorntorp P, Holm J, Schersten T. Metabolic activity of skeletal muscle in patients with peripheral arterial insufficiency. Euro J Clin Invest 1974;4:9-15 
24 Dahllöf AG, Holm J, Schersten T, Sivertsson R. Peripheral arterial insufficiency, effect of physical training on walking tolerance, calf blood flow, and blood flow resistance. Scand J Rehabil Med 1976;8:19-26

25 Degischer S, Labs KH, Hochstrasser J, Aschwanden M, Tschoepl M, Jaeger KA. Physical training for intermittent claudication: a comparison of structured rehabilitation versus home-based training. Vasc Med 2002;7:109-15

26 Gardner AW, Katzel LI, Sorkin JD, Goldberg AP. Effects of long-term exercise rehabilitation on claudication distances in patients with peripheral arterial disease: a randomized controlled trial. J Cardiopulm Rehabil 2002;22:192-8

27 Gelin J, Jivegard L, Taft C, et al. Treatment efficacy of intermittent claudication by surgical intervention, supervised physical exercise training compared to no treatment in unselected randomised patients I: one year results of functional and physiological improvements. Eur J Vasc Endovasc Surg 2001;22: $107-13$

28 Gibellini R, Fanello M, Bardile AF, Salerno M, Aloi T. Exercise training in intermittent claudication. Int Angiol 2000;19:8-13

29 Hiatt WR, Wolfel EE, Meier RH, Regensteiner JG. Superiority of treadmill walking exercise versus strength training for patients with peripheral arterial disease. Implications for the mechanism of the training response. Circulation 1994;90:1866-74

30 Hobbs SD, Marshall T, Fegan C, Adam DJ, Bradbury AW. The constitutive procoagulant and hypofibrinolytic state in patients with intermittent claudication due to infrainguinal disease significantly improves with percutaneous transluminal balloon angioplasty. J Vasc Surg 2006;43:40-6

31 Hobbs SD, Marshall T, Fegan C, Adam DJ, Bradbury AW. The effect of supervised exercise and cilostazol on coagulation and fibrinolysis in intermittent claudication: a randomized controlled trial. J Vasc Surg 2007;45:65-70

32 Hodges LD, Sandercock GR, Das SK, Brodie DA. Randomized controlled trial of supervised exercise to evaluate changes in cardiac function in patients with peripheral atherosclerotic disease. Clin Physiol Funct Imaging 2008;28:32-7

33 Holm J, Dahllöf AG, Bjorntorp P, Schersten T. Enzyme studies in muscles of patients with intermittent claudication. Effect of training. Scand J Clin Lab Invest 1973;128:201-5

34 Izquierdo-Porrera AM, Gardner AW, Powell CC, Katzel LI. Effects of exercise rehabilitation on cardiovascular risk factors in older patients with peripheral arterial occlusive disease. J Vasc Surg 2000;31:670-7

35 Jansen T, Weiss T, Amendt K, Hsu E, Hubsch-Muller C, Diehm C. [Effect of a 2-year ambulatory vascular sports program on walking distance in claudication patients - a controlled study]. Vasa 1991; 33:175

36 Regensteiner JG, Steiner JF, Panzer RJ, Hiatt WR. Evaluation of walking impairment by questionnaire in patients with peripheral arterial disease. J Vasc Med Biol 1990;2:142-52

37 Kakkos SK, Geroulakos G, Nicolaides AN. Improvement of the walking ability in intermittent claudication due to superficial femoral artery occlusion with supervised exercise and pneumatic foot and calf compression: a randomised controlled trial. Eur J Vasc Endovasc Surg 2005;30:164-75

38 Langbein WE, Collins EG, Orebaugh C, et al. Increasing exercise tolerance of persons limited by claudication pain using polestriding. J Vasc Surg 2002;35:887-93

39 Lee HL, Mehta T, Ray B, Heng MS, McCollum PT, Chetter IC. A non-randomised controlled trial of the clinical and cost effectiveness of a Supervised Exercise Programme for claudication. Eur J Vasc Endovasc Surg 2007;33:202-7

40 Mannarino E, Pasqualini L, Innocente S, Scricciolo V, Rignanese A, Ciuffetti G. Physical training and antiplatelet treatment in stage II peripheral arterial occlusive disease: alone or combined? Angiology 1991;42:513-21

41 Mannarino E, Pasqualini L, Menna M, Maragoni G, Orlandi U. Effects of physical training on peripheral vascular disease: a controlled study. Angiology 1989;40:5-10

42 Mika P, Spodaryk K, Cencora A, Mika A. Red blood cell deformability in patients with claudication after pain-free treadmill training. Clin J Sport Med 2006;16:335-40

43 Nielsen SL, Gyntelberg F, Larsen B, Lassen N. Hospital versus home training, a clinical trial. Aktuelle Probleme Angiol 1975;30: $121-6$

44 Nylaende M, Abdelnoor M, Stranden E, et al. The Oslo balloon angioplasty versus conservative treatment study (OBACT) - the 2-years results of a single centre, prospective, randomised study in patients with intermittent claudication. Eur J Vasc Endovasc Surg 2007;33:3-12

45 Patterson RB, Pinto B, Marcus B, Colucci A, Braun T, Roberts M. Value of a supervised exercise program for the therapy of arterial claudication. J Vasc Surg 1997;25:312-8

46 Perkins JM, Collin J, Creasy TS, Fletcher EW, Morris PJ. Exercise training versus angioplasty for stable claudication. Long and medium term results of a prospective, randomised trial. Eur J Vasc Endovasc Surg 1996;11:409-13

47 Regensteiner JG, Meyer TJ, Krupski WC, Cranford LS, Hiatt WR. Hospital vs home-based exercise rehabilitation for patients with peripheral arterial occlusive disease. Angiology 1997;48: 291-300

48 Savage P, Ricci MA, Lynn M, et al. Effects of home versus supervised exercise for patients with intermittent claudication. J Cardiopulm Rehabil 2001;21:152-7

49 Spronk S, Bosch JL, Den Hoed PT, Veen HF, Pattynama PTM, Hunink MG. Clinical effectiveness after endovascular revascularization versus supervised hospital-based exercise training for intermittent claudication: a randomised controlled trial. Radiology 2009;250:586-95

50 Stewart AH, Smith FC, Baird RN, Lamont PM. Local versus systemic mechanisms underlying supervised exercise training for intermittent claudication. Vasc Endovasc Surg 2008;42:314-20

51 Tisi PV, Hulse M, Chulakadabba A, Gosling P, Shearman CP. Exercise training for intermittent claudication: does it adversely affect biochemical markers of the exercise-induced inflammatory response? Eur J Vasc Endovasc Surg 1997;14:344-50

52 Tsai JC, Chan $\mathrm{P}$, Wang $\mathrm{CH}$, et al. The effects of exercise training on walking function and perception of health status in elderly patients with peripheral arterial occlusive disease. J Intern Med 2002;252:448-55

53 Whyman MR, Fowkes FG, Kerracher EM, et al. Is intermittent claudication improved by percutaneous transluminal angioplasty? A randomized controlled trial. J Vasc Surg 1997;26:551-7

54 Mika P, Spodaryk K, Cencora A, Unnithan VB, Mika A. Experimental model of pain-free treadmill training in patients with claudication. Am J Phys Med Rehabil 2005;84:756-62

55 Aquarius AE, Denollet J, Hamming JF, Breek JC, De Vries J. Impaired health status and invasive treatment in peripheral arterial disease: a prospective 1-year follow-up study. J Vasc Surg 2005;41:436-42 
56 Brothers TE, Robison JG, Elliott BM. Prospective decision analysis for peripheral vascular disease predicts future quality of life. J Vasc Surg 2007;46:701-8

57 Collins EG, Langbein WE, Orebaugh C, et al. Cardiovascular training effect associated with polestriding exercise in patients with peripheral arterial disease. J Cardiovasc Nurs 2005;20:177-85

58 Currie IC, Wilson YG, Baird RN, Lamont PM. Treatment of intermittent claudication: the impact on quality of life. Eur J Vasc Endovasc Surg 1995;10:356-61

59 Feinglass J, McCarthy WJ, Slavensky R, Manheim LM, Martin GJ. Functional status and walking ability after lower extremity bypass grafting or angioplasty for intermittent claudication: results from a prospective outcomes study. J Vasc Surg 2000;31:93-103

60 Gardner AW, Katzel LI, Sorkin JD, et al. Exercise rehabilitation improves functional outcomes and peripheral circulation in patients with intermittent claudication: a randomized controlled trial. J Am Geriatr Soc 2001;49:755-62

61 Imfeld S, Singer L, Degischer S, et al. Quality of life improvement after hospital-based rehabilitation or home-based physical training in intermittent claudication. Vasa 2006;35:178-84

62 Pell JP, Lee AJ. Impact of angioplasty and arterial reconstructive surgery on the quality of life of claudicants. The Scottish Vascular Audit Group. Scott Med J 1997;42:47-8

63 Regensteiner JG, Steiner JF, Hiatt WR. Exercise training improves functional status in patients with peripheral arterial disease. J Vasc Surg 1996;23:104-15

64 Taft C, Karlsson J, Gelin J, et al. Treatment efficacy of intermittent claudication by invasive therapy, supervised physical exercise training compared to no treatment in unselected randomised patients II: one-year results of health-related quality of life. Eur J Vasc Endovasc Surg 2001;22:114-23

65 Wann-Hansson C, Hallberg IR, Risberg B, Klevsgard R. A comparison of the Nottingham Health Profile and Short Form 36 Health Survey in patients with chronic lower limb ischaemia in a longitudinal perspective. Health Qual Life Outcomes 2004;2:9

66 Watson L, Ellis B, Leng GC. Exercise for intermittent claudication. Cochrane Database Syst Rev 2008;(4):CD000990

67 Fowkes FG, Gillespie IN. Angioplasty (versus non surgical management) for intermittent claudication. Cochrane Database Syst Rev 2000(2):CD000017

68 Fowkes F, Leng GC. Bypass surgery for chronic lower limb ischaemia. Cochrane Database Syst Rev 2008;(2):CD002000

\section{Appendix 1 - Detailed description of data calculation}

When medians and inter-quartile ranges (IQR) were reported, the mean and SD were estimated by assuming that the median was equal to the mean and the SD was the IQR divided by 1.35 (Cochrane Handbook). ${ }^{18}$

\section{Walking distance}

One trial, Cheetham et al., ${ }^{21}$ did not report standard deviations for walking distances and data were calculated using the reported $P$ values. Dahllöf et al. ${ }^{23}$ and Holm et al. ${ }^{33}$ did not report complete data for the control group. We estimated the walking distances for the control group based on the text and the results for the intervention group. The follow-up means for walking distance of Dahllöf et al. ${ }^{24}$ and Stewart et al. ${ }^{50}$ were calculated using the percentage increase and the mean on baseline. Gibellini et al. ${ }^{28}$ reported the walking distance of the supervised exercise group for symptomatic and asymptomatic patients separately. For these groups, one common mean and standard deviation was calculated. In the study of Larsen et al., ${ }^{8}$ individual patients performed different treadmill tests and for each patient the total amount of consumed calories was calculated and then extrapolated to the walking distance of one treadmill protocol. Mannarino et al. ${ }^{40}$ reported the results of SET versus SET combined with antiplatelet therapy in different groups. For these groups one common mean and standard deviation was calculated and included in the meta-analysis.

\section{Quality of life}

The physical and mental health component were calculated from the eight subscales according to the scoring algorithm, ${ }^{16}$ taking correlations between subscales into account. Five studies only reported parts of the subscales. Component scores were then calculated in a similar way, but using only the available subscales. Spronk et al. ${ }^{49}$ reported all four subscales of the physical health component (physical function, bodily pain, physical role and general health), but no subscales of the mental health component. Currie et al..$^{58}$ reported physical function, bodily pain and physical role, whereas Feinglass et al. ${ }^{59}$ and Imfeld et al. ${ }^{61}$ reported physical function and bodily pain. Cheetham et $a .^{21}$ only reported physical function. Two studies of Regensteiner et al. ${ }^{36,63}$ used the SF-20, reporting five of six subscales (physical function, social function, role functioning, overall health and wellbeing). The subscales physical function and overall health were merged to physical health and social function and wellbeing were merged to form the mental health component. Taft et al. ${ }^{64}$ reported a physical and mental component score of the sickness impact profile. Quality-of-life measurements from Nylaende et al. ${ }^{44}$ and Whyman et al. ${ }^{53}$ using the SF-36 and the Nottingham health profile, respectively, were excluded from the analysis due to lack of data reported in the articles.

\section{Appendix 2 - References of the excluded articles}

1 Albers M, Fratezi AC, De Luccia N. Assessment of quality of life of patients with severe ischemia as a result of infrainguinal arterial occlusive disease. J Vasc Surg 1992;16:54-9 
2 Alpert JS, Larsen OA, Lassen NA. Exercise and intermittent claudication. Blood flow in the calf muscle during walking studied by the xenon-133 clearance method. Circulation 1969;39:353-9

3 Andreozzi GM, Leone A, Laudani R, Martin R, Deinit G, Cataldi V. Levo-propionyl-carnitine improves the effectiveness of supervised physical training on the absolute claudication distance in patients with intermittent claudication. Angiology 2008;59:84-9

4 Aquarius AE, Denollet J, de Vries J, Hamming JF. Poor health-related quality of life in patients with peripheral arterial disease: type D personality and severity of peripheral arterial disease as independent predictors. J Vasc Surg 2007;46:507-12

5 Aquarius AE, Denollet J, Hamming JF, Van Berge Henegouwen DP, De Vries J. Type-D personality and ankle brachial index as predictors of impaired quality of life and depressive symptoms in peripheral arterial disease. Arch Surg 2007;142:662-7

6 Back MR, Kluess HA, Huber TS, et al. Evaluation of skeletal muscle metabolic responses following exercise training in patients with intermittent claudication. Vasc Surg 2000;34:345-59

7 Bonde-Petersen F, Siggaard-Andersen J. Physical performance capacity in patients with dysbasia arteriosclerotica. V. Physical performance capacity and flow capacity in the single legs as a limiting factor in two-leg exercise tests. Scand J Rehabil Med 1974;6:41-5

8 Bosch JL, van der Graaf Y, Hunink MG. Health-related quality of life after angioplasty and stent placement in patients with iliac artery occlusive disease: results of a randomized controlled clinical trial. The Dutch Iliac Stent Trial Study Group. Circulation 1999;99:3155-60

9 Bosch JL, Halpern EF, Gazelle GS. Comparison of preferencebased utilities of the Short-Form 36 Health Survey and Health Utilities Index before and after treatment of patients with intermittent claudication. Med Decis Making 2002;22:403-9

10 Boyd CE, Bird PJ, Teates CD, Wellons HA, MacDougall MA, Wolfe LA. Pain free physical training in intermittent claudication. J Sports Med Phys Fitness 1984;24:112-22

11 Bracht CHR, Schmidt JA, Pölnitz AV, Höfling B. Transkutaner sauerstoffdruck am fuss unter laufbandbelastung vor und nach perkutaner atherektomie. Vasa 1992;21:258-62

12 Buck M, Ciccone CD. Does evidence exist on whether specific interventions can improve adherence to a home exercise program in a patient with intermittent claudication? Phys Ther 2004;84: 465-73

13 Cachovan M, Scheffler P, Gruss J, Diehm C, Rogatti W. [The effectiveness of standardized exercise training in intermittent claudication]. Wien Klin Wochenschr 1994;106:517-20

14 Carmeli E, Barchad S, Masharawi Y, Coleman R. Impact of a walking program in people with down syndrome. J Strength Cond Res 2004;18:180-4

15 Clifford PC, Davies PW, Hayne JA, Baird RN. Intermittent claudication: is a supervised exercise class worth while? $B M J$ 1980;280:1503-5

16 Coffman JD. Intermittent claudication--be conservative. $N$ Engl J Med 1991;325:577-8

17 Collins EG, Langbein WE, Orebaugh C, et al. Polestriding exercise and vitamin $\mathrm{E}$ for management of peripheral vascular disease. Med Sci Sports Exerc 2003;25:384-93

18 Collins TC, Krueger PN, Kroll TL, Sharf BF. Face-to-face interaction compared with video watching on use of physical activity in peripheral arterial disease: a pilot trial. Angiology 2009; 60:21-30

19 Collins TC, Johnson SL, Souchek J. Unsupervised walking therapy and atherosclerotic risk-factor management for patients with peripheral arterial disease: a pilot trial. Ann Behav Med 2007;33:318-24

20 Creasy TS, McMillan PJ, Fletcher EW, Collin J, Morris PJ. Is percutaneous transluminal angioplasty better than exercise for claudication? Preliminary results from a prospective randomised trial. Eur J Vasc Endovasc Surg 1990;4:135-40

21 Deutschmann HA, Schoellnast H, Temmel W, et al. Endoluminal therapy in patients with peripheral arterial disease: prospective assessment of quality of life in 190 patients. AJR Am J Roentgenol 2007;188:169-75

22 de Vries M, Ouwendijk R, Kessels AG, et al. Comparison of generic and disease-specific questionnaires for the assessment of quality of life in patients with peripheral arterial disease. $J$ Vasc Surg 2005;41:261-8

23 Dotter CT, Judkins MP. Transluminal treatment of arteriosclerotic obstruction. Description of a new technic and a preliminary report of its application. Circulation 1964;30:654-70

24 Drozdz W, Panek J, Lejman W. Red cell deformability in patients with chronic atheromatous ischemia of the legs. Med Sci Monit 2001;7:933-9

25 Elson JD, Raymond RA. Lower extremity ischemia. Interventions to preserve quality of life. Postgrad Med 1994;95:96-100, 103-8

26 Ericsson B, Haeger K, Lindell SE. Effect of physical training of intermittent claudication. Angiology 1970;21:188-92

27 Ernst EE, Matrai A. Intermittent claudication, exercise, and blood rheology. Circulation 1987;76:1110-4

28 Ernst E. Peripheral vascular disease. Benefits of exercise. Sports Med 1991;12:149-51

29 Fernandes e Fernandes J, Nicolaides AN, Angelides NA, GordonSmith IC. An objective assessment of common femoral endarterectomy and profundaplasty in patients with superficial femoral occlusion. Surgery 1978;83:313-8

30 Fowler B, Jamrozik K, Norman P, Allen Y, Wilkinson E. Improving maximum walking distance in early peripheral arterial disease: randomised controlled trial. Aust J Physiother 2002;48: 269-75

31 Fujioka K, Esato K, Zempo N, Katoh T, Fujimura Y, Yoshimura $\mathrm{K}$. Arterial reconstruction: justified for patients with intermittent claudication? World J Surg 1998;22:1039-42

32 Galea MN, Bray SR, Ginis KA. Barriers and facilitators for walking in individuals with intermittent claudication. J Aging Phys Act 2008;16:69-83; quiz 84

33 Gardner AW, Killewich LA, Montgomery PS, Katzel LI. Response to exercise rehabilitation in smoking and nonsmoking patients with intermittent claudication. J Vasc Surg 2004;39:531-8

34 Gardner AW, Montgomery PS, Flinn WR, Katzel LI. The effect of exercise intensity on the response to exercise rehabilitation in patients with intermittent claudication. $J$ Vasc Surg 2005;42:702-9

35 Gruntzig A, Hopff $\mathrm{H}$. [Percutaneous recanalization after chronic arterial occlusion with a new dilator-catheter (modification of the Dotter technique) (author's transl)]. Dtsch Med Wochenschr 1974;99:2502-10, 2511

36 Hedberg B, Langstrom $M$, Angquist KA, Fugl-Meyer AR. Isokinetic plantar flexor performance and fatiguability in peripheral arterial insufficiency. Effects of training vs. vascular surgery. Acta Chir Scand 1988;154:363-9

37 Heinrich F. [Improvement of walking disability and conservative treatment. Guiding principles for drug treatment of chronic arterial occlusive disease (stage I and IIb)]. Fortschr Med 1984;102: $74-5$ 
38 Hiatt WR, Regensteiner JG, Hargarten ME, Wolfel EE, Brass EP. Benefit of exercise conditioning for patients with peripheral arterial disease. Circulation 1990;81:602-9

39 Hiatt WR, Regensteiner JG, Wolfel EE, Carry MR, Brass EP. Effect of exercise training on skeletal muscle histology and metabolism in peripheral arterial disease. J Appl Physiol 1996;81: $780-8$

40 Hosie KB, Kockelberg R, Newbury-Ecob RA, Callum KG, Nash JR. A retrospective review of the outcome of patients over 70 years of age considered for vascular reconstruction in a district general hospital. Eur J Vasc Surg 1990;4:313-5

41 Hoynck van Papendrecht AA, van Bemmelen SP. [Treatment of patients with intermittent claudication with walking exercises]. NTvG 1986;130:1360-4

42 Janzon L, Bergentz SE, Ericsson BF, Lindell SE. Intermittent claudication and hypertension. Ankle pressure and walking distance in patients with well-treated and non-treated hypertension. Angiology 1981;32:175-9

43 Johnson BF, Singh S, Evans L, Drury R, Datta D, Beard JD. A prospective study of the effect of limb-threatening ischaemia and its surgical treatment on the quality of life. Eur J Vasc Endovasc Surg 1997;13:306-14

44 Jonason T, Jonzon B, Ringqvist I, Oman-Rydberg A. Effect of physical training on different categories of patients with intermittent claudication. Acta Med Scand 1979;206:253-8

45 Jonason T, Ringqvist I, Oman-Rydberg A. Home-training of patients with intermittent claudication. Scand J Rehabil Med 1981;13:137-41

46 Jones PP, Skinner JS, Smith LK, John FM, Bryant CX. Functional improvements following StairMaster vs. treadmill exercise training for patients with intermittent claudication. J Cardiopulm Rehabil 1996;16:47-55

47 Kiesewetter H, Blume J, Jung F, Gerhards M, Leipnitz G. [Training by walking and drug therapy of peripheral arterial occlusive disease]. Vasa 1987;20:384-7

48 Kiesewetter H, Jung F, Blume J, Bulling B, Gerhards M. Conservative drug therapy and walking exercise in stage IIb peripheral arterial occlusion disease. Klin Wochenschr 1986;64:1061-9

49 Killewich LA. Improving functional status and quality of life in elderly patients with peripheral arterial disease. J Am Coll Surg 2006;202:345-55

50 Klevsgard R, Froberg BL, Risberg B, Hallberg IR. Nottingham Health Profile and Short-Form 36 Health Survey questionnaires in patients with chronic lower limb ischemia: before and after revascularization. J Vasc Surg 2002;36:310-7

51 Klevsgard R, Hallberg IR, Risberg B, Thomsen MB. The effects of successful intervention on quality of life in patients with varying degrees of lower-limb ischaemia. Eur J Vasc Endovasc Surg 2000;19:238-45

52 Larsen OA, Lassen NA. Medical treatment of occlusive arterial disease of the legs. Walking exercise and medically induced hypertension. Angiologica 1969;6:288-301

53 Linhart J, Vanek I. Treatment of intermittent claudication: two different mechanisms. Int Angiol 1991;10:6-9

54 Lundgren F, Dahllöf AG, Schersten T, Bylund-Fellenius AC. Muscle enzyme adaptation in patients with peripheral arterial insufficiency: spontaneous adaptation, effect of different treatments and consequences on walking performance. Clin Sci (Lond) 1989;77:485-93

55 Maass U, Cachovan M, Alexander K. [Effect of interval training on walking distance, hemodynamics and ventilation in patients with intermittent claudication. II. Changes of hemodynamics and ventilation]. Vasa 1983;12:326-32

56 Manfredini F, Malagoni AM, Mascoli F, et al. Training rather than walking: the test in-train out program for home-based rehabilitation in peripheral arteriopathy. Circ J 2008;72:946-52

57 McDermott MM, Tiukinhoy S, Greenland $\mathrm{P}$, et al. A pilot exercise intervention to improve lower extremity functioning in peripheral arterial disease unaccompanied by intermittent claudication. J Cardiopulm Rehabil 2004;24:187-96

58 Mehta T, Venkata Subramaniam A, Chetter I, McCollum P. Assessing the validity and responsiveness of disease-specific quality of life instruments in intermittent claudication. Eur J Vasc Endovasc Surg 2006;31:46-52

59 Mika P, Spodaryk K, Cencora A, Unnithan VB, Mika A. Experimental model of pain-free treadmill training in patients with claudication. Am J Phys Med Rehabil 2005;84:756-62

60 Mori E, Komori K, Kume M, Yamaoka T, Shoji T, Furuyama T, Inoguchi $\mathrm{H}$. Comparison of the long-term results between surgical and conservative treatment in patients with intermittent claudication. Surgery 2002;131:S269-74

61 Myhre K, Sorlie DG. Physical activity and peripheral atherosclerosis. Scand J Soc Med 1982;29:195-201

62 Nawaz S, Walker RD, Wilkinson CH, Saxton JM, Pockley AG, Wood RF. The inflammatory response to upper and lower limb exercise and the effects of exercise training in patients with claudication. J Vasc Surg 2001;33:392-9

63 Nylaende M, Kroese AJ, Morken B, et al. Beneficial effects of 1-year optimal medical treatment with and without additional PTA on inflammatory markers of atherosclerosis in patients with PAD. Results from the Oslo Balloon Angioplasty versus Conservative Treatment (OBACT) study. Vasc Med 2007;12: 275-83

64 Peabody CN, Kannel WB, McNamara PM. Intermittent claudication. Surgical significance. Arch Surg 1974;109:693-7

65 Petersen FB. The effects of varying walking speeds when measuring the claudication distance on horizontal and sloping levels. Acta Chir Scand 1967;133:627-30

66 Reifler DR, Feinglass J, Slavensky R, Martin GJ, Manheim L, McCarthy WJ. Functional outcomes for patients with intermittent claudication: bypass surgery versus angioplasty versus noninvasive management. J Vasc Med Biol 1994;5:203-11

67 Sanderson B, Askew C, Stewart I, Walker P, Gibbs H, Green S. Short-term effects of cycle and treadmill training on exercise tolerance in peripheral arterial disease. J Vasc Surg 2006;44: $119-27$

68 Saxton JM, Zwierska I, Hopkinson K, et al. Effect of upper- and lower-limb exercise training on circulating soluble adhesion molecules, hs-CRP and stress proteins in patients with intermittent claudication. Eur J Vasc Endovasc Surg 2008;35:607-13

69 Scheffler P, de la Hamette D, Gross J, Mueller H, Schieffer H. Intensive vascular training in stage IIb of peripheral arterial occlusive disease. The additive effects of intravenous prostaglandin E1 or intravenous pentoxifylline during training. Circulation 1994;90:818-22

70 Scheffler P, de la Hamette D, Muller H. [Controlled vascular training in IIb peripheral arterial occlusive disease: additive effect of intravenous PGE1 versus intravenous pentoxifylline during training]. Vasa 1991;33:350-2

71 Schlussel H. [Exercise training in older patients with chronic peripheral vascular disease]. Munch Med Wochenschr 1965;107: $1772-5$ 
72 Schlussel H. [Walking exercise in the interval of chronic occlusion of the leg arteries]. Arch Phys Ther (Leipz) 1966;18:313-7

73 Schoop W. Mechanism of beneficial action of daily walking training of patients with intermittent claudication. Scand J Clin Lab Invest 1973;128:197-9

74 Schutz RM. [Exercise therapy in peripheral arterial occlusive disease in advanced age-a sensible measure?] Z Gerontol 1988;21:327-9

75 Spronk S, Bosch JL, den Hoed PT, Veen HF, Pattynama PM, Hunink MM. Cost-effectiveness of endovascular revascularization compared to supervised hospital-based exercise training in patients with intermittent claudication: a randomized controlled trial. J Vasc Surg 2008;48:1472-80

76 Spronk S, Dolman W, Boelhouwer RU, Veen HF, den Hoed PT. The vascular nurse in practice: Results of prescribed exercise training in patients with intermittent claudication. J Vasc Nurs 2003;21:141-4

77 Taft C, Sullivan M, Lundholm K, Karlsson J, Gelin J, Jivegard L. Predictors of treatment outcome in intermittent claudication. Eur J Vasc Endovasc Surg 2004;27:24-32

78 Tennesen KH. Exercise blood flow by 133-xenon clearance in claudicating patients: reproducibility and sensitivity of the method in comparison to angiography, venous occlusion plethysmography, and walking distance on escalator. Scand J Clin Lab Invest 1967;100:84

79 Tonnesen KH. Muscle blood flow during exercise in intermittent claudication. Validation of the 133-xenon clearance technique: clinical use by comparison to plethysmography and walking distance. Circulation 1968;37:402-10

80 Tse RL. Daily walking exercise in treatment of intermittent claudication: it works. Angiology 1971;22:1-3

81 Ubels FL, Links TP, Sluiter WJ, Reitsma WD, Smit AJ. Walking training for intermittent claudication in diabetes. Diabetes Care 1999;22:198-201

82 Walker RD, Nawaz S, Wilkinson CH, Saxton JM, Pockley AG, Wood RF. Influence of upper- and lower-limb exercise training on cardiovascular function and walking distances in patients with intermittent claudication. J Vasc Surg 2000;31:662-9

83 Wang E, Hoff J, Loe H, Kaehler N, Helgerud J. Plantar flexion: an effective training for peripheral arterial disease. Eur J Appl Physiol 2008;104:749-56

84 Wang J, Zhou S, Bronks R, Graham J, Myers S. Effects of supervised treadmill walking training on calf muscle capillarization in patients with intermittent claudication. Angiology 2009;60: 36-41

85 Wang J, Zhou S, Bronks R, Graham J, Myers S. Effects of supervised treadmill-walking training on strength and endurance of the calf muscles of individuals with peripheral arterial disease. Clin J Sport Med 2006;16:397-400

86 Wilson SE, Sheppard B. Results of percutaneous transluminal angioplasty for peripheral vascular occlusive disease. Ann Vasc Surg 1990;4:94-7

87 Whyman MR, Fowkes FG, Kerracher EM, et al. Randomised controlled trial of percutaneous transluminal angioplasty for intermittent claudication. Eur J Vasc Endovasc Surg 1996;12: $167-72$

88 Whyman MR, Ruckley CV, Fowkes FG. Angioplasty for mild intermittent claudication. Br J Surg 1991;78:643-5

89 Williams LR, Ekers MA, Collins PS, Lee JF. Vascular rehabilitation: benefits of a structured exercise/risk modification program. J Vasc Surg 1991;14:320-6

90 Willigendael EM, Bendermacher BL, Olde Damink SWM, Welten RJ, Prins MH, Teijink JA. Exercise therapy for patients with peripheral arterial disease; the value of an accelerometer to register physical activity during two therapy regimes; a pilot randomised clinical trial. In: The Care for Peripheral Arterial Disease, A Multidisciplinary Approach. Dordrecht: University of Maastricht, 2005

91 Winterfeld HJ, Siewert $\mathrm{H}$, Strangfeld D, Schmidt HH. [Experiences with the application of physiotherapy in peripheral arterial circulatory disorders of the lower extremities (stage I and IIa) under ambulatory conditions]. Z Gesamte Inn Med 1983;38:221-5

92 Wolf GL, Wilson SE, Cross AP, Deupree RH, Stason WB. Surgery or balloon angioplasty for peripheral vascular disease: a randomized clinical trial. Principal investigators and their Associates of Veterans Administration Cooperative Study Number 199. J Vasc Interv Radiol 1993;4:639-48

93 Wolosker N, Nakano L, Rosoky RA, Puech-Leao P. Evaluation of walking capacity over time in 500 patients with intermittent claudication who underwent clinical treatment. Arch Intern Med 2003;163:2296-300

94 Wood RE, Sanderson BE, Askew CD, Walker PJ, Green S, Stewart IB. Effect of training on the response of plasma vascular endothelial growth factor to exercise in patients with peripheral arterial disease. Clin Sci (Lond) 2006;111:401-9

95 Zetterquist $\mathrm{S}$. The effect of active training on the nutritive blood flow in exercising ischemic legs. Scand J Clin Lab Invest 1970; 25:101-11

96 Zwierska I, Walker RD, Choksy SA, Male JS, Pockley AG, Saxton JM. Upper- vs lower-limb aerobic exercise rehabilitation in patients with symptomatic peripheral arterial disease: a randomized controlled trial. J Vasc Surg 2005;42:1122-30 